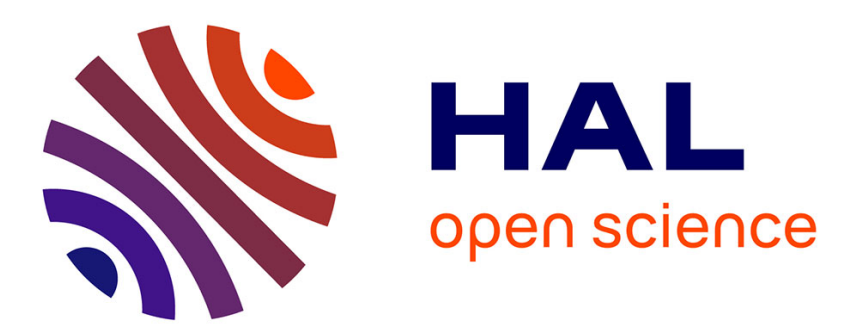

\title{
Numerical Stability issues on Channelized Hotelling Observer under different background assumptions
} Di Ge, Lu Zhang, Christine Cavaro-Ménard, Patrick Le Callet

\section{To cite this version:}

Di Ge, Lu Zhang, Christine Cavaro-Ménard, Patrick Le Callet. Numerical Stability issues on Channelized Hotelling Observer under different background assumptions. Journal of the Optical Society of America. A Optics, Image Science, and Vision, 2014, 31 (5), pp.1112-1117. inserm-00986008

\section{HAL Id: inserm-00986008 https://www.hal.inserm.fr/inserm-00986008}

Submitted on 30 Apr 2014

HAL is a multi-disciplinary open access archive for the deposit and dissemination of scientific research documents, whether they are published or not. The documents may come from teaching and research institutions in France or abroad, or from public or private research centers.
L'archive ouverte pluridisciplinaire HAL, est destinée au dépôt et à la diffusion de documents scientifiques de niveau recherche, publiés ou non, émanant des établissements d'enseignement et de recherche français ou étrangers, des laboratoires publics ou privés. 


\title{
Numerical Stability issues on Channelized Hotelling Observer under different background assumptions
}

\author{
Di Ge, ${ }^{1}$ Lu Zhang, ${ }^{2, *}$ Christine Cavaro-Ménard, ${ }^{2}$ and Patrick Le Callet ${ }^{3}$ \\ ${ }^{1}$ LTSI, INSERM UMR 1099, University of Rennes 1, Bat. 22, 35042 Rennes Cedex, France \\ ${ }^{2}$ LISA, EA 4094, University of Angers, 62 avenue Notre Dame du Lac, 49000 Angers, France \\ ${ }^{3}$ IRCCyN, UMR-6597, University of Nantes, Rue Christian Pauc, BP50609, 44306 Nantes cedex 3, France
}

\begin{abstract}
This paper addresses the numerical stability issue on the Channelized Hotelling Observer (CHO). The CHO is a well-known approach in the medical image quality assessment domain. Many researchers have found that the detection performance of the CHO does not increase with the number of channels, contrary to expectation. And to our knowledge, nobody in this domain has found the reason. We illustrated that this is due to the ill-posed problem of the scatter matrix and proposed a solution based on Tikhonov regularization. Although Tikhonov regularization has been used in many other domains, we show in this paper another important application of Tikhonov regularization. This is very important for researchers to continue the CHO (and other channelized model observer) investigation with a reliable detection performance calculation.
\end{abstract}

\section{INTRODUCTION}

The necessity and the importance of objective image quality measurement has long been acknowledged in the medical imaging community [1-4]. To this end, numerical observers are used [5-7] to predict the outcome of a human observer performing certain diagnostic tasks (e.g., detection, localization). Their usefulness relies on the approximation to the performances achieved by human observers and in particular by clinical experts. Studies of numerical observers are largely motivated by their being less expensive and cumbersome than human observers.

The detection task is a binary classification with two underlying hypotheses (presence or absence of a signal in the image). The ideal observer (IO) was firstly proposed for the optimization of the binary classification problem, by choosing the hypothesis resulting in the greatest likelihood of the image data. The IO achieves the maximum AUC (area under the Receiver Operating Characteristic curve) among the observers, but the computation of likelihoods are tractable only for simplified cases. The Hotelling Observer (HO, aka Optimum Linear Discriminant) on the other hand maximizes the SNR among all linear observers while using only the first and secondorder statistics of the image data, i.e., the mean and the covariance matrix of the vectorized image data. The inversion of the (large) covariance matrix and its accurate estimate are the main difficulties in practical applications. To avoid the inversion problem of big matrix, data reduction method based on Channelized Hotelling Observers (CHO) was proposed to approach the SNR performance of the $\mathrm{HO}$ under sufficient statistics conditions [8]. The channel design is also a mathematical model (based on filter banks $[9,10]$ ) to approach the human perceptual and cognitive behaviors.

Despite its simplicity in numerical implementation and flexibility in channel design to incorporate human per-

\footnotetext{
* Corresponding author: lu.ge@insa-rennes.fr
}

ception models, the $\mathrm{CHO}$ yields counter intuitive results when it is implemented blindly. Increasing the number of channels could worsen the detection performance, making it difficult to choose the optimal channel number with respect to human experts' diagnoses. mip is a present study, we investigate the cause of the numerical instability and find that it is due to the direct inversion method applied to ill-conditioned scatter matrices. We propose a regularization method well established in the world of linear algebra for the $\mathrm{CHO}$ implementation and its performances are shown to be more stable while increasing channel numbers. To illustrate the numerical instability and the efficiency of our proposed regularization method, simulation tests are performed on signal known exactly (SKE) detection task in which all the signal attributes (e.g. intensity amplitude, size, shape, orientation and location) are known and do not vary throughout the experiment.

\section{PROBLEM FORMULATION}

We describe briefly here the CHO in the SKE detection task and the derivation of its scalar test statistic. Specific attentions are given to the problem of performance instability w.r.t. increasing channel numbers. The detection problem is considered as the validation of one of the two exclusive hypotheses : $\mathcal{H}_{0}$ (signal absent) and $\mathcal{H}_{1}$ (signal present). The observed image data $\boldsymbol{g}$ is given by:

$$
\mathcal{H}_{h}: \boldsymbol{g}=h \boldsymbol{x}+\boldsymbol{b}, \quad h=0,1
$$

where the known signal is denoted by $\boldsymbol{x}$, the background by $\boldsymbol{b}$, and the absence or the presence of the signal is controlled by the binary variable $h$. In the following, a 2D image (test image data, signal or background) is represented by a column vector using vertical concatenation. Let the observed image have $\sqrt{M} \times \sqrt{M}$ pixels, its vectorized version $\boldsymbol{g}$ is then a $M \times 1$ vector. 


\section{A. Signal model and CHO}

The current work considers the basic detection task of a rotationally symmetric nodule signal profile in image quality assessment and the image generation process follows [6]. The background correlation structure and the imaging system are both rotationally symmetric.

We use the 2D Gaussian signal model proposed in [11, 12] :

$$
[\boldsymbol{x}]_{\boldsymbol{p}}=a \exp \left(-\frac{1}{2}(\boldsymbol{p}-\boldsymbol{q})^{\mathrm{t}} \mathbf{D}^{-1}(\boldsymbol{p}-\boldsymbol{q})\right)
$$

where both $\boldsymbol{p}$ and $\boldsymbol{q}$ are 2 -dimensional column vectors containing the sampling grid index. $[\boldsymbol{x}]_{p}$ denotes the signal intensity at $\boldsymbol{p}$. The signal intensity attenuation is hereby modeled by a Gaussian function of peak amplitude $a$, centered at $\boldsymbol{q}$. The diagonal matrix $\mathbf{D}$ such that $\operatorname{diag}(\mathbf{D})=\left[b \sigma^{2}, \sigma^{2}\right]^{\mathrm{t}}$ specifies the ellipse's scale $\sigma$ and shape $b(b=1$ in the spheric case). Note that when all signal parameters are known exactly in advance by observers, the detection task is said to be a SKE task.

Four artificial background models are mostly used in the literature: white Gaussian background, correlated Gaussian background, lumpy background [13], and clustered lumpy background [14]. Note that all of these backgrounds are rotationally symmetric in the statistical sense. The superposition of the signal and the background yields simulated test images, presented in Fig. 1.
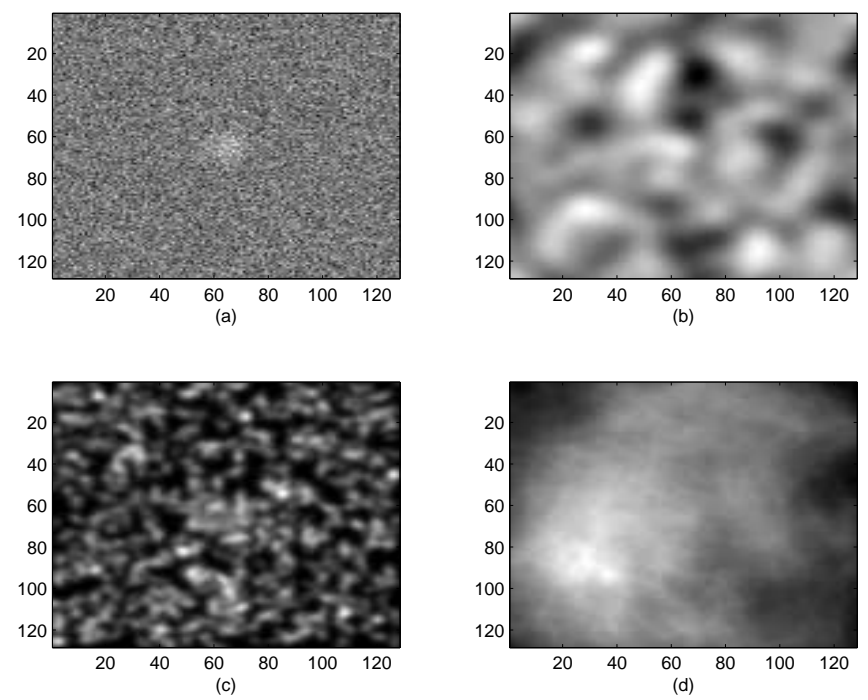

FIG. 1. Simulated test images $(128 \times 128)$ with gaussian lesion profile in the center of different backgrounds: (a) White Gaussian, (b) Correlated Gaussian, (c) Lumpy, (d) Clustered lumpy.

The CHO pre-processes an image by channelization (dimension reduction by matrix multiplication):

$$
\boldsymbol{g}^{\prime}=\mathbf{U}^{\mathrm{t}} \boldsymbol{g}
$$

for which $\mathbf{U}_{M \times p}$ contains $p$ channel $(p \ll M)$ responses in each column. All calculations afterwards are per- formed on the channelized data, denoted by $(\cdot)^{\prime}$ in the following.

In practice, the SKE CHO can be implemented by a training stage and a test stage. In the training stage, two sets of image are considered : signal absent and signal present. The output template writes :

$$
\boldsymbol{w}_{\mathrm{CHO}}=\widehat{\mathbf{S}}^{-1} \widehat{\boldsymbol{x}}^{\prime}
$$

using channelized signal and scatter matrix estimate $\left(\widehat{\boldsymbol{x}^{\prime}}\right.$ and $\widehat{\mathbf{S}}^{\prime}$ respectively) :

$$
\begin{aligned}
& \widehat{\boldsymbol{x}^{\prime}}=\overline{\boldsymbol{g}_{1}}{ }^{\prime}-\overline{\boldsymbol{g}}_{0}{ }^{\prime}, \\
& \widehat{\mathbf{S}^{\prime}}=\frac{1}{2}\left(\operatorname{Var}\left(\boldsymbol{g}_{1}^{\prime}\right)+\operatorname{Var}\left(\boldsymbol{g}_{0}^{\prime}\right)\right)
\end{aligned}
$$

The empirical means and covariances are noted as ${ }^{-}$and $\operatorname{Var}(\cdot)$ respectively while the subscript $\cdot_{0}$ and $\cdot_{1}$ denote the signal class of the input signal. An inversion of the $p \times p$ matrix $\widehat{\mathbf{S}^{\prime}}$ in Eq. (4) is needed.

In the test stage, the above-trained template $\boldsymbol{w}_{\mathrm{CHO}}$ is used to calculate a test statistic $\lambda_{C H O}$ linearly w.r.t. $\boldsymbol{g}^{\prime}$ :

$$
\lambda_{\mathrm{CHO}}=\boldsymbol{w}_{\mathrm{CHO}}^{\mathrm{t}} \boldsymbol{g}^{\prime}
$$

A figure of merit can be calculated in order to characterize the CHO detection performance, such as :

$$
\mathrm{SNR}=\frac{\left(\overline{\lambda_{0}}-\overline{\lambda_{1}}\right)^{2}}{\left(\operatorname{Var}\left(\lambda_{0}\right)+\operatorname{Var}\left(\lambda_{1}\right)\right) / 2}
$$

where test statistics are obtained for both classes $\left(\lambda_{0}\right.$ and $\lambda_{1}$ respectively). Another widely used figure of merit for binary classifier (AUC) can be obtained by summing the area under the ROC (receiver operating characteristic) curve. In the following, we choose to show graphically the performance vs number of channels using the SNR measure since it is more capable of quantifying the separation of the two classes if the measure of the AUC is sufficiently close to unity, as argued in [15]. Note the two measures are equivalent if the test statistics follow the independent and identically distributed (i.i.d.) Gaussian distributions [3]. Gallas et al. [8] has shown that the CHO maximizes the SNR among all linear observers with reduced dimensions under the sufficient statistics conditions, i.e., channels should be chosen to effectively represent the $\mathrm{HO}$ template.

\section{B. CHO simulation results}

Gallas et al. [8] has proposed the Laguerre-Gauss (LG) channels to adapt to the rotational symmetric properties of the signal and background model. The LG functions are the product of Laguerre polynomials and Gaussian (exponential) weightings. By combining the Gaussian decay, the LG functions in polar coordinates writes:

$$
u_{n}\left(\boldsymbol{r} \mid a_{U}\right)=\frac{\sqrt{2}}{a_{U}} \exp \left(-\frac{\pi \boldsymbol{r}^{2}}{a_{U}^{2}}\right) L_{n}\left(\frac{2 \pi \boldsymbol{r}^{2}}{a_{U}^{2}}\right)
$$


where $\boldsymbol{r} \in \mathbb{R}^{+}$is the radial distance variable and $a_{u}$ the spread parameter of the LG channel. These functions are then sampled and vectorized to form the column vectors $U_{n}$ of $\mathbf{U}$ in Eq. (3). It is argued in [8] that an optimal choice of the parameter $a_{U}$ is related to the spread of the signal and the correlation length of the lumpy background. 2-D LG functions in Eq. (9) are calculated with different values of $a_{U}$ for a $128 \times 128$ image and are shown in Fig. 2. L-G channels having smaller $a_{U}$ tend to have more local effects.
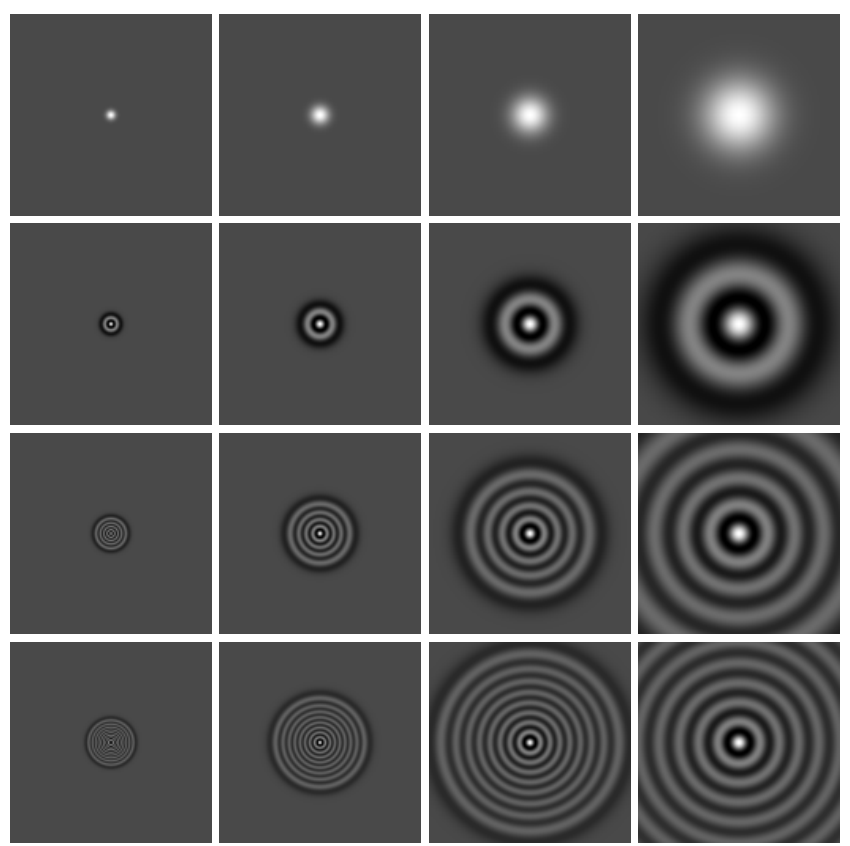

FIG. 2. LG functions for $a_{U}=5,10,20,40$ (column) and the order of channel $=0,3,9,17$ (line). The grayscale is normalized by the factor of $\frac{\sqrt{2}}{a_{U}}$ for each column.

Performances of $\mathrm{CHO}$ detection using direct inverse of the scatter matrix (cf Eq. (4)) are shown in Fig. 3. L-G channel numbers vary from 2 to 40 while ranges of the spread parameter $a_{u}$ are adjusted to include the optimal value in each case. Two Gaussian signal profiles (with $\sigma=2$ and $\sigma=5$ respectively) are tested over the four backgrounds in Fig. 1. In all cases, 2000 training images and another 2000 testing images are generated in simulation. forming a sufficient databse to acurately estimate the inverse covariance matrix considering the learning curve by Based upon this simple simulation dataset, we show the difficulties in explaining test results of classical $\mathrm{CHO}$ implementations if the number of channels increases.

While the white Gaussian background allows relative performance stability for all L-G channels, the detection performances do not necessarily improve by increasing the channel numbers for the other background sets. On the contrary, important downgrades are frequently observed in Fig. 3 (2)-(4) and this is a general result since no particular channel numbers do worse than others. However it is expected that by increasing the channel numbers
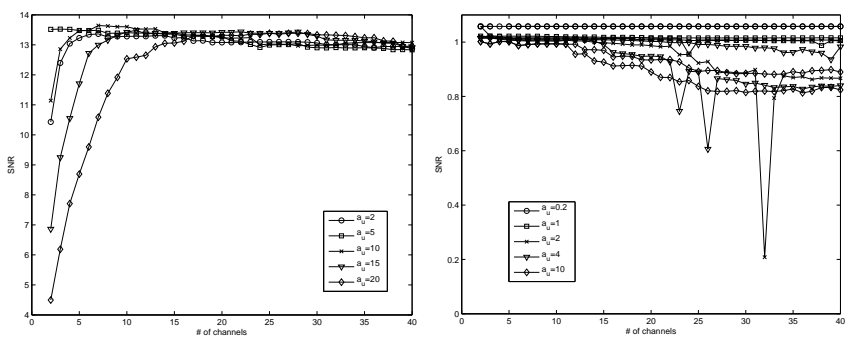

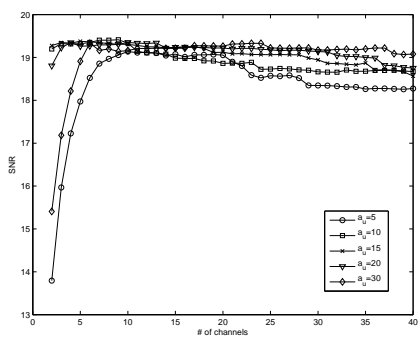

(1) White Gaussian background with profile $\sigma=2$ (upper) and $\sigma=5$ (lower)
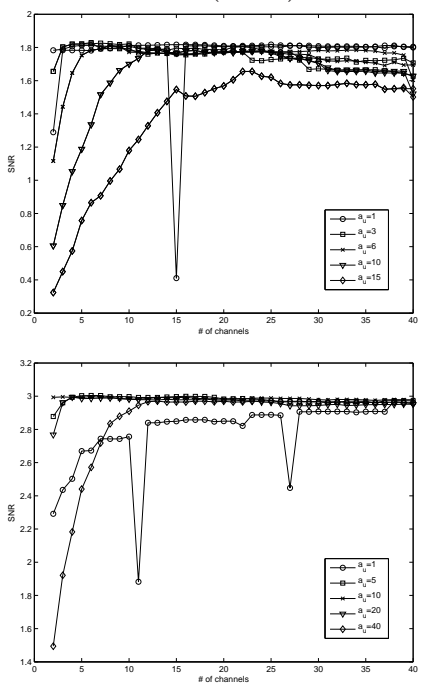

(3) Lumpy background with profile $\sigma=2$ (upper) and $\sigma=5$ (lower)

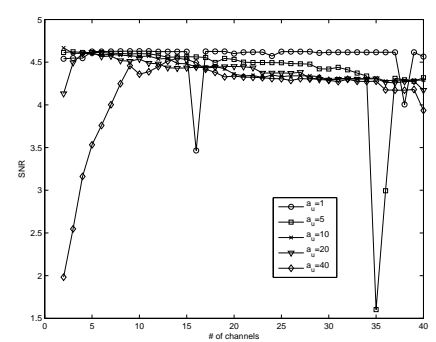

(2) Correlated Gaussian background with profile $\sigma=2$ (upper) and $\sigma=5$ (lower)
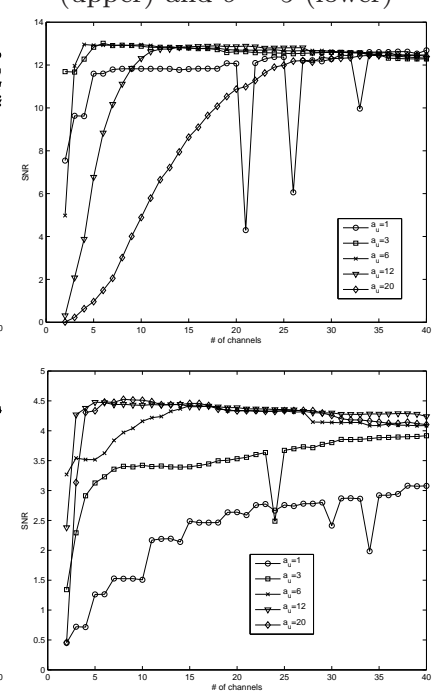

(4) Clustered lumpy background with profile $\sigma=2$ (upper) and $\sigma=5$ (lower)
FIG. 3. L-G CHO detection performances (SNR) using different L-G channel numbers with direct inverse (by Gaussian elimination) of the scatter covariance matrix $\widehat{\mathbf{S}^{\prime}}$. Four backgrounds are tested, each with 2000 training image inputs and 2000 testing image. L-G spread parameters $a_{u}$ (c.f. Eq. (9)) and the $y$-axis ranges are adjusted for each case. The Channel numbers all range from 2 to 40 .

an asymptote in the figure of merit should correspond to the performance of the ideal linear observer, i.e., without any dimension reduction [8]. The problem is that the inverse of the covariance matrix $\widehat{\mathbf{S}}^{-1}$ in Eq. (6) is not accurately estimated since more channels require more cases, as was demonstrated by Chan et al.[16] and in some cases it may not even be invertible (see discussion on $a_{u}$ in the next section). While L-G channels with bigger $a_{u}$ tend to 
yield more stable performances in general, in some cases such as Fig. 3 (2) smaller $a_{u}$ could have interesting potentials if it were not for the stability issue. Note the zoom-in effect of the y-axis for each scenario in Fig. 3.

This result is particularly frustrating since the L-G channels are chosen because continuous L-G functions form a basis on the space of rotationally symmetric square-integrable functions in $\mathbb{R}^{2}$ : any rotational symmetric function $y(\boldsymbol{r})$ can be exactly expanded by :

$$
y(\boldsymbol{r})=\sum_{n=0}^{\infty} \alpha_{n} u_{n}\left(\boldsymbol{r} \mid a_{U}\right)
$$

with $\alpha_{n}$ the inner product defined by :

$$
\alpha_{n}=\frac{a_{U}}{\sqrt{2}} \int y(\boldsymbol{r}) u_{n}\left(\boldsymbol{r} \mid a_{U}\right) \mathrm{d} \boldsymbol{r} .
$$

It is expected that the more channels are applied, the better detection performance should be achieved.

We investigate in the following the numerical cause and propose a method accordingly. Thell the as any modelobservers are doveloped to be comparable to human observers and not to surpass them in performances. It is worth noting that the motivation of this study is the maximization of the detection performance of the L-G CHO, and by doing so achieve a stabilized relationship w.r.t. the channel numbers so that the choice of parameters like $\left\{p, a_{U}\right\}$ based on the comparison with human observers' performances is unbiased.

\section{MATRIX CONDITION AND INVERSION}

To our knowledge, no previous study has been made to achieve reasonable detection performances w.r.t. increasing channel numbers even though it is necessary priorly to the determination of optimal channel parameter. Indeed, the inversion of the covariance matrix $\left(\widehat{\mathbf{S}^{\prime}}\right)$ becomes more sensitive to the spread parameter and finite sample size if more channels are added (curse of dimensionality). Chan et al. [16] studied the effect of finite sample size on classifier designs for computer-aided diagnosis (CAD) algorithms and showed empirically the linear dependences between the AUC and the reciprocal of the number of training samples. Generally speaking, the sample size available for classifier design is limited and thus introduces variance and bias into the detection performance. In the current study, we show that the "dimension curse" is arriving more rapidly than expected with small spread parameters (cf Fig. 4), such that increasing the number of training images to achieve better estimate of the inverse covariance matrix is no longer an option : either unrealistic in the case of medical imaging or too much time consuming in simulation tests.

Apart from the sample number issue, the real covariance matrix itself could not be invertible (ill-posed). The L$\mathrm{G}$ channels are not a basis on a sampled grid. What is worse, these channels become almost linearly dependent when the $a_{u}$ parameter is pushed to smaller values due to the sampling grid effect (see the aliasing for the case $a_{u}=5, p=17$ in Fig. 2). This is typically what's happening in Fig. 4 with $a_{u} \leq 1$ : the condition numbers of the covariance matrix (the ratio between the biggest eigenvalue in absolute value and that of the smallest) start with high levels and are extremely fast growing afterwards. We also observe that :

1. the condition numbers grow exponentially by increasing $p$ of L-G channels for all spread parameters;

2. they run quickly out of the dynamic range $\left(10^{20}\right)$ given the float point precision $\epsilon=10^{-16}$ of the simulation settings, making them extremely illconditioned matrices $\left(\operatorname{cond}(\cdot) \gg \epsilon^{-1}\right)$;

3. larger $a_{U}$ yields slower increase but remains exponential. However, smaller $a_{U}$ might have better performances in some cases (cf Fig. 3) if stabilized, which makes the regularized solution inevitable if we aim to optimize the $\mathrm{CHO}$ performances;

4. the other backgrounds yield similar results as that of the lumpy background case in Fig. 4.

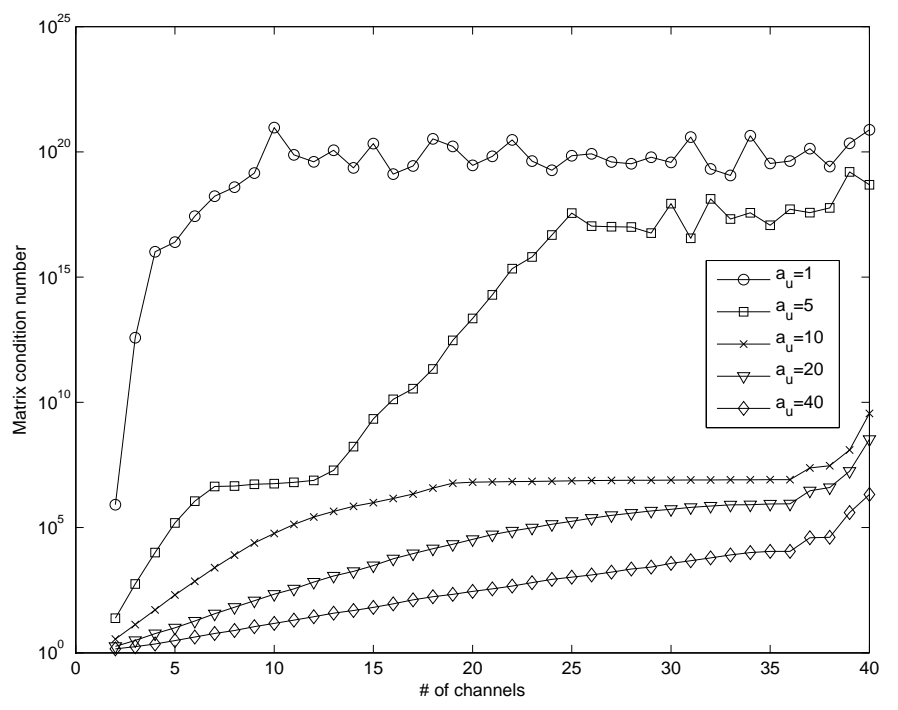

FIG. 4. Example of the condition number of the matrix $\widehat{\mathbf{S}^{\prime}}$ with increasing dimension $p$ for the five classes of L-G channels $\left(a_{u}=\left[\begin{array}{lllll}1 & 5 & 10 & 20 & 40\end{array}\right]\right)$. The test is realized on a lumpy background identical to the lower panel (signal profile $\sigma=5$ ) of Fig. 3(3)

Higher order L-G channels are thus penalized by the fast growing condition number of the covariance matrix estimate in $\mathrm{CHO}$ applications. It is also interesting to note that without further adaptations, it is reasonable to privilege L-G channels with higher values for the spread parameter $a_{U}$ because of their numerical stability of the inverse covariance matrix $\widehat{\mathbf{S}^{\prime}}$. However, as shown in Fig. 3 and argued in [8] its optimal value is dependent on the 
spread of the known signal $s$ and background correlation properties. This is also the reason why this numerical instability issue should be addressed so that the choice of the optimal $a_{U}$ and the performances vs channel number curves are unbiased. In the following, we propose a numerical fix to achieve a more meaningful curve for the figure of merit, the latter being the prerequisite to finding the optimal $\mathrm{CHO}$ model w.r.t. the human perceptual and cognitive tasks [9].

\section{REGULARIZED INVERSION}

The empirical estimate matrix $\widehat{\mathbf{S}^{\prime}}$ is by construction (cf Eq. (6)) non-negative definite and allows the following SVD decomposition :

$$
\widehat{\mathbf{S}}^{\prime}=\mathbf{V D V}^{\mathrm{t}}
$$

where $\mathbf{V}$ is an orthonormal matrix containing normalized eigenvectors $\left(\mathbf{V} \mathbf{V}^{\mathrm{t}}=\mathbf{I}\right)$, and $\mathbf{D}=\operatorname{diag}\left(d_{1}, \ldots, d_{p}\right)$ is a diagonal matrix whose main diagonal values are eigenvalues in the descending order $d_{1} \geq \ldots \geq d_{p}>0$. We recall that the condition number is the ratio $d_{1} / d_{p}$. The direct inverse writes ${\widehat{\mathbf{S}^{\prime}}}^{-1}=\mathbf{V D}^{-1} \mathbf{V}^{\mathrm{t}}$.

The Tikhonov regularization is particularly adapted to the evaluation of moderate dimension and ill-conditioned problems such as Eq. (4) and provides a direct closedform solution. Instead of calculating Eq. (4) by direct inverse as the solution of a linear problem :

$$
\widehat{\mathbf{S}^{\prime}} \cdot \boldsymbol{w}=\widehat{\boldsymbol{x}^{\prime}}
$$

a bias is introduced deliberately by considering the following problem :

$$
\min \left\{\frac{1}{2}\left\|\widehat{\mathbf{S}^{\prime}} \cdot \boldsymbol{w}-\widehat{\boldsymbol{x}^{\prime}}\right\|^{2}+\frac{1}{2} \eta\|\boldsymbol{w}\|^{2}\right\}, \quad \eta>0
$$

for which the positive coefficient $\eta$ controls the regularization level of the solution. From the SVD point of view, the regularization method can be applied to replace ${\widehat{\mathbf{S}^{\prime}}}^{-1}$ in Eq. (4) with $\boldsymbol{w}_{\mathrm{CHO}}^{\dagger}=\mathbf{S}^{\dagger} \widehat{\boldsymbol{x}}^{\prime}$ where

$$
\mathbf{S}^{\dagger}=\left({\widehat{\mathbf{S}^{\prime}}}^{\mathrm{t}} \widehat{\mathbf{S}}^{\prime}+\eta \mathbf{I}\right)^{-1}{\widehat{\mathbf{S}^{\prime}}}^{\mathrm{t}}=\mathbf{V}\left[\begin{array}{ccc}
\frac{d_{1}}{\eta+d_{1}^{2}} & & \\
& \ddots & \\
& & \frac{d_{p}}{\eta+d_{p}^{2}}
\end{array}\right] \mathbf{V}^{\mathrm{t}}
$$

and $\eta$ is chosen such that $d_{1}^{2} \gg \eta \gg d_{p}^{2}$ for the extremely ill-conditioned cases. We note that for $\widehat{\mathbf{S}}^{\prime}$ with relatively small condition numbers, the regularization has negligible effects by imposing $d_{1}^{2} \gg \eta: \mathbf{S}^{\dagger}$ is close to the direct inverse of $\widehat{\mathbf{S}^{\prime}}$ and thus the detection performances do not change significantly. On the contrary, for extremely illconditioned cases the presence of $\eta$ avoids the division by zero. Indeed, the regularization technique attempts to achieve numerical stability by penalizing the template norm $\left\|\boldsymbol{w}_{\mathrm{CHO}}\right\|^{2}$ so that the latter would not grow out of control as in the case of the direct inverse by Eq. (4). Evidently, bigger $\eta$ values could further regularize the template solution norm, at the cost of a more important bias.

We present in Fig. 5 the figure of merit (SNR) for the detection performances on the simulated data used in Fig. 3. Detection performances are more smoothly increasing if not strictly monotonic. Most importantly, the regularized inversion method stabilizes L-G channels with relatively small spread parameters that in some cases are the optimal L-G CHO (cf the lower panel of Fig. 5(2) and the upper panel of Fig. 5(3)).
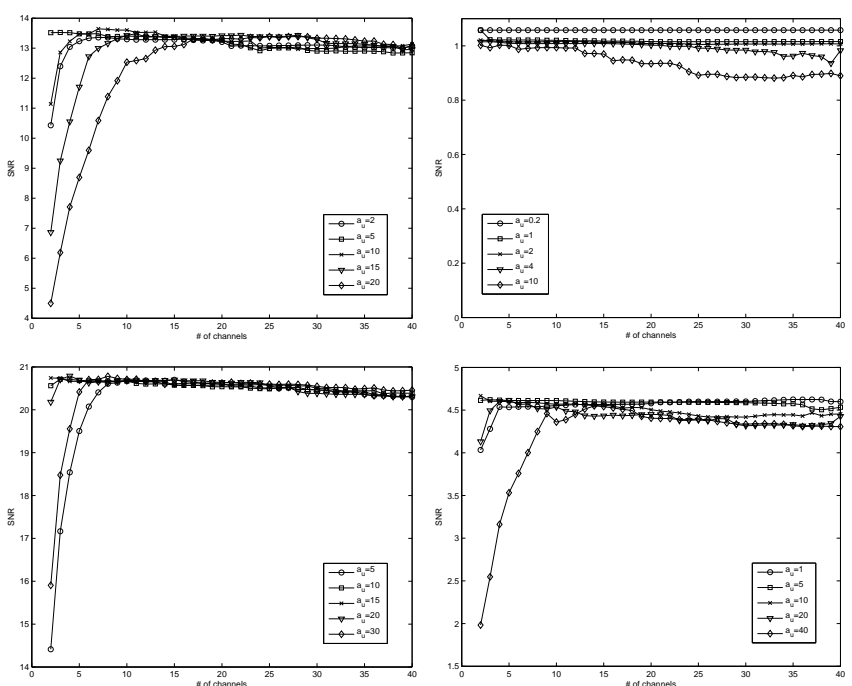

(1) White Gaussian background with profile $\sigma=2$ (upper) and

(2) Correlated Gaussian

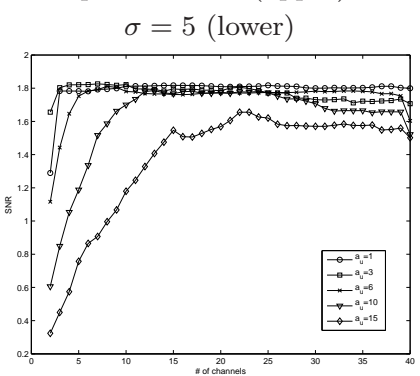
background with profile $\sigma=2$ (upper) and $\sigma=5$ (lower)
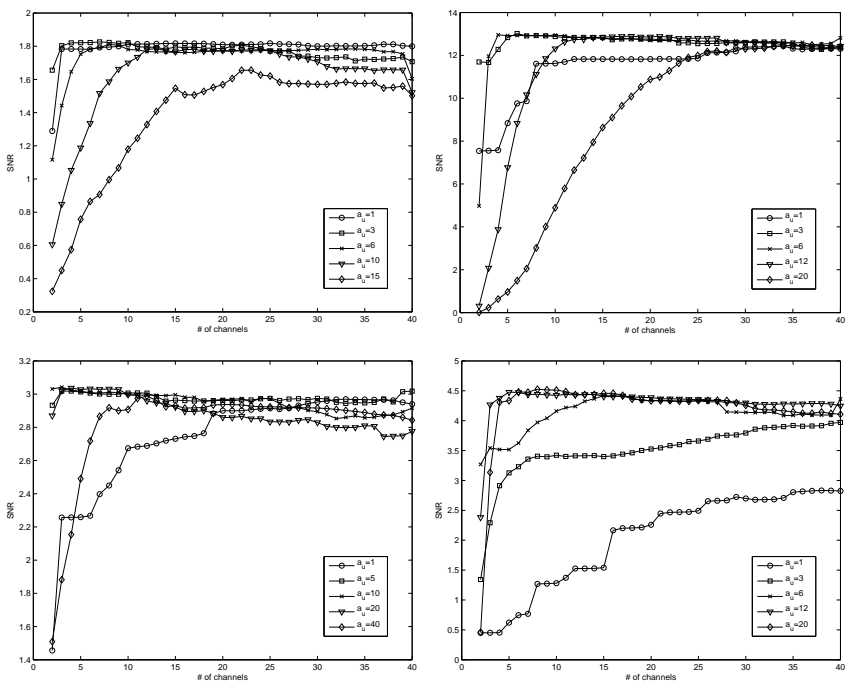

(3) Lumpy background with profile $\sigma=2$ (upper) and $\sigma=5$ (lower)

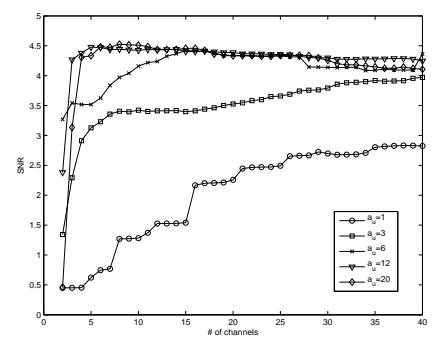

(4) Clustered lumpy background with profile $\sigma=2$ (upper) and $\sigma=5$ (lower)

FIG. 5. SNR with proposed Tikhonov regularization for all backgrounds with simulation data identical to Fig. 3. The $\mathrm{y}$-axis ranges are adjusted for each case given specific backgrounds and signal sizes. 


\section{CONCLUSION}

We presented in the current study the idea of using Tikhonov regularization method to address the extremely ill-conditioned scatter matrix inversion problem, the main cause of the numerical instability observed in the SKE detection tasks. Rotationally symmetric backgrounds and signals are chosen together with adapted L-G channels to illustrate the principle. It is indeed a generic problem : other channels such as the DoG channels and the Gabor channels that have shown capable of closely tracking human performances are equally subject to the phenomenon. Our goal is to achieve stable performance results with respect to the channel numbers for a given dataset such that the choice of optimal parameters should not be influenced. In that sense, the Tikhonov regularization method achieves that goal by obtaining a stable performance asymptote that characterizes the upper limit of linear observers.
[1] H. H. Barrett, "Objective assessment of image quality: Effects of quantum noise and object variability," JOSA A 7, 1266-1278 (1990).

[2] H. H. Barrett, J. L. Denny, R. F. Wagner, and K. J. Myers, "Objective assessment of image quality. II. Fisher information, Fourier crosstalk, and figures of merit for task performance," JOSA A 12, 834-852 (1995).

[3] H. H. Barrett, C. K. Abbey, and E. Clarkson, "Objective assessment of image quality. III. ROC metrics, ideal observers, and likelihood-generating functions," JOSA A 15, 1520-1535 (1998).

[4] J. P. Rolland and H. H. Barrett, "Effect of random background inhomogeneity on observer detection performance," JOSA A 9, 649-658 (1992).

[5] T. Narayan and G. Herman, "Prediction of human observer performance by numerical observers: an experimental study." JOSA A 13, 679-693 (1999).

[6] C. K. Abbey and H. H. Barrett, "Human- and modelobserver performance in ramp-spectrum noise: effects of regularization and object variability," JOSA A 18, 47348 (2001).

[7] P. Bruyant, H. Gifford, G. Gindi, P. Pretorius, and M. King, "Human and numerical observer studies of lesion detection in ga-67 images obtained with map-em reconstructions and anatomical priors," in "NSS/MIC," , vol. 7 (2004), vol. 7, pp. 4072-4075.

[8] B. D. Gallas and H. H. Barrett, "Validating the use of channels to estimate the ideal linear observer," JOSA A 20, 1725-1738 (2003).

[9] S. Daly, The visible differences predictor: an algorithm for the assessment of image fidelity (Cambridge, MA: MIT Press, 1993), chap. 14, pp. 179-206.
[10] A. Watson and J. Solomon, "Model of visual contrast gain control and pattern masking," JOSA A 14, 2379-91 (1997).

[11] L. Zhang, C. Cavaro-Ménard, P. L. Callet, and L. H. K. Cooper, "The effects of anatomical information and observer expertise on abnormality detection task," in "in Proc. SPIE Medical Imaging," , vol. 7966 (2011), vol. 7966.

[12] B. Goossens, L. Platiša, E. Vansteenkiste, and W. Philips, "The use of steerable channels for detecting asymmetrical signals with random orientations," in "SPIE Medical Imaging," (International Society for Optics and Photonics, 2010), pp. 76270S-76270S.

[13] S. Park, "Performance of a channelized-ideal observer using laguerre-gauss channels for detecting a gaussian signal at a known location in different lumpy backgrounds," in "Proceedings of SPIE," , vol. 6146 (Spie, 2006), vol. 6146, pp. 61460P-61460P-9.

[14] C. Castella, K. Kinkel, F. Descombes, M. Eckstein, P. Sottas, F. Verdun, and F. Bochud, "Mammographic texture synthesis: second-generationclustered lumpy backgrounds using agenetic algorithm," Opt. Express 16, 7595-7607 (2008).

[15] S. Park, A. Badano, B. D. Gallas, and K. J. Myers, "Incorporating Human Contrast Sensitivity in Model Observers for Detection Tasks," IEEE Trans. Med. Imag. 28, 339-347 (2009).

[16] H.-P. Chan, B. Sahiner, R. F. Wagner, and N. Petrick, "Classifier design for computer-aided diagnosis: Effects of finite sample size on the mean performance of classical and neural network classifiers," Medical Physics 26, 2654-68 (1999). 